\title{
Scattering of dipole-mode vector solitons: Theory and experiment
}

\author{
Wieslaw Krolikowski ${ }^{1}$, Glen McCarthy ${ }^{1}$, Yuri S. Kivshar ${ }^{2}$, Carsten Weilnau ${ }^{3}$, Cornelia Denz ${ }^{3}$, \\ Juan J. García-Ripoll ${ }^{4}$, and Víctor M. Pérez-García ${ }^{4}$ \\ ${ }^{1}$ Laser Physics Center, Research School of Physical Sciences and Engineering, \\ The Australian National University, Canberra, ACT 0200, Australia \\ ${ }^{2}$ Nonlinear Physics Group, Research School of Physical Sciences and Engineering, \\ The Australian National University, Canberra, ACT 0200, Australia \\ 3 Institute of Applied Physics, Westfälische Wilhelms Universität Münster, D-48149 Münster, Germany \\ ${ }^{4}$ Departamento de Matemáticas, Universidad de Castilla-La Mancha, $130{ }^{r 1}$ Ciudad Real, Spain
}

\begin{abstract}
We study, both theoretically and experimentally, the scattering properties of optical dipole-mode vector solitons - radially asymmetric composite self-trapped optical beams. First, we analyze the soliton collisions in an isotropic two-component model with a saturable nonlinearity and demonstrate that in many cases the scattering dynamics of the dipole-mode solitons allows us to classify them as "molecules of light" - extremely robust spatially localized objects which survive a wide range of interactions and display many properties of composite states with a rotational degree of freedom. Next, we study the composite solitons in an anisotropic nonlinear model that describes photorefractive nonlinearities, and also present a number of experimental verifications of our analysis.
\end{abstract}

PACS numbers: 42.65.Tg, 05.45.Yv, 47.20.Ky

\section{INTRODUCTION}

An understanding of the interaction of simple physical objects leading to the formation of more complex objects is an ultimate goal of fundamental research in many fields of physics. Recent progress in generating spatial optical solitons - the self-trapped states of light with particlelike properties - in various nonlinear bulk media allows to study the truly two-dimensional self-trapping of light and different types of interaction of multi-dimensional solitary waves, including the formation of more complicated localized states [1].

Spatial optical solitons have attracted considerable attention as possible building blocks of all-optical switching devices where light is used to guide and manipulate light itself [1]. The robust nature of spatial optical solitons displayed in the propagation and interaction [2] allows us to draw an analogy with atomic physics, treating spatial solitons as "atoms of light". Furthermore, when several light beams generated by coherent sources are combined to produce vector or composite solitons, this process can be viewed as the formation of composite states or "molecules of light".

Recently, the existence of robust "molecules of light" in the form of dipole-mode vector solitons was predicted theoretically [3] and also verified experimentally [4]. The dipole-mode solitons (or 'dipoles', for simplicity) originate from the trapping of a dipole-mode optical beam by an effective waveguide created by a mutually incoherent fundamental beam of nearly radial symmetry. The first observation of this novel type of optical vector soliton was reported in Ref. [4], where the dipoles were generated using two different methods: the phase imprinting technique and a symmetry-breaking instability of a vortex-mode composite soliton, another type of fundamental (radially symmetric) composite soliton created by the incoherent coupling of two optical beams $[5,6]$. It is worth to mention that vector solitons can be also created in certain processes involving coherent interaction of waves such as second harmonic generation. In this particular situation, the two constituent beams forming a soliton molecule are fundamental and its second harmonic, respectively [7].

The concept of vector solitons as 'molecules of light' can be compared with photonic microcavity structures, micrometer-size "photonic quantum dots" that confine photons in such a way that they act like electrons in an atom [8]. When two of these "photonic atoms" are coupled together, they produce a "photonic molecule" whose optical modes bear a strong resemblance to the electronic states in a diatomic molecule like hydrogen [9]. The selftrapped states of light we study here can be viewed as somewhat similar photonic structures where, the photonic trap and the beam it guides are both made of light and create self-trapped photonic atoms and molecules.

In this paper we present a comprehensive study of the scattering properties of the dipole-mode vector solitons and analyze, in particular, the interaction between these objects and other self-trapped structures such as scalar optical solitons and other dipoles. We describe a number of interesting effects observed in numerical simulations of such interactions, for both isotropic saturable and anisotropic nonlocal nonlinear models. These include the absorption of a soliton by a dipole and the replacement of the soliton with a dipole component, the field momentum redistribution that can be viewed as the transformation of a linear momentum into an angular momentum with the subsequent dipole spiraling, etc. Additionally, we verify experimentally some of our analytical predictions by studying the generation and scattering of the composite spatial solitons in photorefractive nonlinear crystals. The versatility of the phenomena described here makes dipole-mode vector solitons of great importance not only because of the fundamental interest in nonlinear physics 
but also because of potential promising applications in all optical switching and integrated optics.

The paper is organized as follows. Section II studies the scattering of scalar solitons and dipole-mode vector solitons in the framework of an isotropic saturable nonlinear medium. In this section, we also present the most important examples of the dipole-soliton interactions. Section III includes the studies of the formation and interaction of the dipole-mode solitons in an anisotropic nonlocal nonlinear model which is used for describing the nonlocal anisotropic nonlinearities of photorefractive media. In Sec. IV, we summarize the results of our experimental studies of the interaction of the dipole solitons in photorefractive nonlinear crystals. Finally, Sec. V concludes the paper.

\section{SOLITON SCATTERING IN A SATURABLE ISOTROPIC MEDIUM}

\section{A. Model and solitons}

We consider here the propagation of two light beams interacting incoherently in a saturable nonlinear medium. In the steady state regimes in the paraxial approximation, the mutual beam interaction can be described by a system of two coupled nonlinear Schrödinger (NLS) equations [3, 4, 5, 10],

$$
\begin{aligned}
& i \frac{\partial u}{\partial z}=-\frac{1}{2} \triangle_{\perp} u+F(I) u, \\
& i \frac{\partial v}{\partial z}=-\frac{1}{2} \triangle_{\perp} v+F(I) v,
\end{aligned}
$$

where $u\left(\mathbf{r}_{\perp}, z\right)$ and $v\left(\mathbf{r}_{\perp}, z\right)$ are the dimensionless envelopes of the beams which are self-trapped in the crosssection plane $\mathbf{r}_{\perp}=(x, y)$ and propagate along the direction $z$. The function $F(I)=I(1+s I)^{-1}$ characterizes a saturable nonlinearity of the medium, where $s$ is a dimensionless saturation parameter $(0<s<1)$ and $I=|u|^{2}+|v|^{2}$ is the total beam intensity.

We would like to mention that we consider here only the stationary propagation of light excluding any nonlinearity-mediated temporal effects. In fact, the experimental observations indicate that this is a common situation in many nonlinear systems involving free (no feedback) propagation of optical beams in both fast (such as atomic) and slow (photorefractive, thermal) nonlinear media. Typically, the only dynamics which may occur in such cases is related to switching effects and dies out within the time scale determined by the temporal response of the medium. However, the temporal in noninstantaneous media such as photorefractive crystals may lead to a number of novel transiting effects (see, e.g., Refs. 11, 12, 13, 14]).

Equations (11) describe different types of spatially localized composite solutions. The dipole-mode vector soliton (or "a molecule of light") is a stationary state which is composed of a nodeless beam in the $v$ component and a dipole beam (or a pair of out-of-phase solitons) in the $u$ component. Solitons in the $u$ component have opposite phases and thus they repel each other, but the role of the complimentary beam $v$ is to stabilize the structure making it robust. A numerical analysis of the linearized equations (11) shows no signs of linear instability of this composite structure [3], as was also recently confirmed by means of the asymptotic analytical theory [15]. Moreover, it was shown [3] that such robust dipolemode vector solitons exist for a wide range of the beam powers $P_{u}=\int|u|^{2} d \mathbf{r}_{\perp}$ and $P_{v}=\int|v|^{2} \mathbf{r}_{\perp}$. Since we are interested in showing stability far from the regime in which one beam is dominant, all numerical experiments are performed using as initial conditions stationary states in which $P_{u} \simeq P_{v}$.

We are interested in the dynamics of the dipole soliton under the action of finite external perturbations introduced by its collision with other objects. The word "finite" emphasizes the fact that we can no longer make use of linearized equations and that we must deal with the full system (1). This fact, combined with the complex structure of the dipole which lacks radial symmetry, makes analytical predictions on the dipole dynamics very difficult. Nevertheless, as will be shown below, one may extract some general rules on which qualitative predictions may be based.

The idea is that the dipole can be seen as a bound state of a soliton beam (in $v$ ) plus a pair of vortices with opposite charges (in $u$ ) and, therefore, many of the effects observed in the composite beam collisions and described below can be understood once the mutual interaction of these simpler objects is studied.

One of the components of the dipole is a soliton beam (to be referred to as soliton hereafter). Spatial solitons are stable localized states which have no nodes and which are the states of minimum energy of the system for a fixed power. When two of these solitons are in different, mutually incoherent beams (say, one in $u$ and the other one, in $v$ ), they interact incoherently and attract each other. Thus, during an incoherent interaction two solitons may either become bound or scatter. In the former case, we have an example of what we call a molecule of light, which is typically referred to as a "vector soliton". However, when two solitons are derived from the same beam they interact coherently and the outcome of their mutual interaction depends on their phase difference. When this quantity is small or zero, solitons experience mutual attraction, whereas if their mutual phases differ by $\pi$, they repel each other.

Another nonlinear structure that should be mentioned in this context is a vortex-mode composite soliton introduced in [5] which in our model (11) is stable only in the vicinity of the bifurcation point 15]. Thus, the vortices may only be stabilized by co-propagating with a very large soliton beam (e.g., when a vortex in the linear beam $u$ is guided by an effective waveguide created in the component $v$ ). Otherwise, a composite state of a vortex plus a soliton constitutes an unstable molecule of light. 
A dipole can be seen as a pair of vortices as described above or, alternatively, as a bound state of two solitons with a phase difference of $\pi$. While in principle, these solitons should repel each other, the system is stabilized due to the interaction with a soliton-induced waveguide created by the other, mutually incoherent, component.

\section{B. Numerical results for the soliton collisions}

\section{Soliton-dipole scattering}

The first type of numerical simulations we present here consists in shooting a scalar soliton against a dipole-mode vector soliton. All the simulations discussed here have been performed using a split-step operator technique using FFT, with grid sizes of up to $512 \times 512$ points covering a rectangular domain of $68 \times 34$ adimensional units. The initial data are always a combination of stationary states. For instance, when a soliton is launched against a dipole, we start with

$$
\begin{aligned}
& u(\mathbf{x}, 0)=u_{\text {dipole }}(\mathbf{x})+u_{\text {soliton }}(\mathbf{x}-\mathbf{d}) e^{-i \mathbf{p}_{\mathbf{0}} \mathbf{x}}, \\
& v(\mathbf{x}, 0)=v_{\text {dipole }}(\mathbf{x})
\end{aligned}
$$

Here $\mathbf{d}=\left(d_{x}, d_{y}\right), d_{x} \gg d_{y}, d_{y}$ is the impact parameter, and $\mathbf{p}_{\mathbf{0}}$ is proportional to the initial (linear) momentum of the incoming scalar soliton. The initial data $u_{\text {dipole }}$, $u_{\text {soliton }}$, and $v_{\text {dipole }}$ are obtained numerically by a suitable minimization procedure outlined in Ref. 3].

The result is an inelastic collision in which the soliton becomes deflected and the dipole gains both linear and angular momenta. The whole process is depicted in Fig.1. Soliton scattering occurs when the incident beam has medium to large linear momentum or when it has an appropriate initial phase. For instance, in Fig.1 the incident soliton has sign (-) and it crashes against the part of the dipole with $(+)$ sign. A conservation law forces the dipole to rotate and the soliton becomes deflected, sometimes as much as by a 90 degree angle.

When the linear momentum of the incident soliton is large, it moves too fast to suffer a destructive influence from the dipole. In Fig. 2 we plot the exchange of the linear momentum between the soliton and dipole as a function of the impact parameter. The effective interaction is clearly attractive: the soliton coming from below $\left(d_{y}<0\right)$ feels the drag of the dipole above it and gets deflected upwards $\left(p_{y}>0\right)$, while the dipole moves downwards.

The second family of numerical experiments is performed with solitons which are slow and, as is usual in scattering processes, the effects of the interaction process may be more drastic. For some impact parameters the soliton gets too close to the lobe of the dipole with the smallest phase difference and fuses with it with some emission of radiation and a subsequent rotation of the dipole. This is well reflected in Fig.3 (radiation is not seen).

\section{Dipole-dipole collisions}

The third family of numerical simulations corresponds to shooting dipoles against each other. These collisions, which resemble interaction of atomic molecules provide a rich source of phenomena depending on the mutual orientation of the dipoles and on the initial energy. Figure 4 summarizes the main results observed. There we see three cases (a-c) in which the dipole solitons are preserved. The figure shows an in-phase collision with weak interaction [Fig.4(a)], an out-of-phase collision with repulsion [Fig.4(b)], and an example of the collision with nonzero impact parameter in which two vortex states are created and they decay into a pair of spiralling solitons [Fig.4(c)].

The last case, Fig.4 (d), shows an interesting inelastic process when two dipoles fuse into a more complex state which then decays creating a new dipole and a pair of simple solitons. All these processes may be understood in terms of the phase of the lobes of each dipole as described above.

\section{SOLITON SCATTERING IN ANISOTROPIC NONLOCAL MEDIA}

\section{A. Composite solitons}

The dipole-mode vector solitons considered so far were restricted to those realized in isotropic nonlinear media. However, up to now the majority of experimental observations of dipole-mode and multi-pole vector solitons have been performed in photorefractive nonlinear crystals which are known to exhibit anisotropy in their nonlinear response [16]. In effect, even circularly symmetric optical beams induce strongly asymmetric refractive index changes which significantly affect the formation of spatial solitons as well as their interaction.

In this section we employ the commonly accepted model for the photorefractive nonlinearity that takes into account its most important properties [17] to investigate some of the previously discussed examples of interactions of vector solitons.

The interactions we consider here involve dipole-mode vector solitons and scalar solitons. The dipole-mode vector solitons consist of two mutually incoherent optical beams with the envelopes $u$ and $v$, propagating in a bulk anisotropic nonlocal nonlinear medium such as a biased photorefractive crystal. When the characteristic spatial scales are larger than the photorefractive Debye length and the diffusion field may be neglected, the steady-state propagation along the $z$ axis of a photorefractive crystal with an externally applied electric field along the $x$ axis 
is described by the equations:

$$
\begin{aligned}
& i \frac{\partial u}{\partial z}+\frac{1}{2} \nabla^{2} u=-\frac{\gamma}{2} \frac{\partial \varphi}{\partial x} u, \\
& i \frac{\partial v}{\partial z}+\frac{1}{2} \nabla^{2} v=-\frac{\gamma}{2} \frac{\partial \varphi}{\partial x} v, \\
& \nabla^{2} \varphi+\nabla \varphi \nabla \ln (1+I)=E_{0} x_{0} \frac{\partial}{\partial x} \ln (1+I),
\end{aligned}
$$

where $\gamma$ and $E_{0}$ are the normalized nonlinearity coefficient and external field, respectively, $I \equiv|u|^{2}+|v|^{2}$ is the total intensity, $\nabla=\hat{x}(\partial / \partial x)+\hat{y}(\partial / \partial y)$, and $\varphi$ is the dimensionless electrostatic potential induced by the light with the boundary condition $\nabla \varphi(\vec{r} \rightarrow \infty) \rightarrow 0$. The propagation coordinate $z$ is measured in units of the diffraction length, and the transverse coordinates are normalized by the characteristic beam size $x_{0}$. The above system of equations was solved numerically by applying concurrently the finite difference and split-step Fast Fourier methods to the electrostatic potential and propagation equations, respectively and using a 256x256 grid size computational window.

\section{B. Soliton-dipole scattering}

The first two of the numerical simulations we present here consist of colliding a scalar soliton into one lobe of a dipole-mode vector soliton. The scalar soliton is coherent with the dipole component of the vector soliton and can thus be considered part of the same beam in the theoretical model (4) above. The initial data for all cases presented in this section reflect the experimental conditions in that they are not exact solutions to the model. The fundamental component of the vector soliton and the scalar beam are Gaussian beams and the dipole component is the first derivative of a Gaussian beam along $y$ coordinate.

The first example of the collision between the dipolemode vector soliton and the scalar soliton is shown in Fig.5. Here the scalar soliton is in phase with the lobe of the dipole with which it collides. This leads to a strong attraction between the scalar beam and the lobe and eventual absorption of the former followed by, as in the isotropic case, a rotation of the whole dipole-mode vector soliton. In a photorefractive crystal the complete rotation of a dipole soliton is prohibited by the anisotropy of the nonlinear refractive index change [18]. Hence, unlike the isotropic case (see Fig.1), the vector soliton exhibits only angular oscillations about the vertical axis. As Fig.5 clearly shows the collision leads to dipole soliton experiencing also a lateral shift which is due to transfer of a linear momentum from the scalar soliton to the vector soliton. As our numerical simulations show the outcome of this collision can be more dramatic if the intensity of the scalar beam is increased. In such a case the collision may result in the break up of the vector soliton such that the two out-of-phase lobes of the dipole are no longer trapped. Such an effect occurs when the intensity of the scalar beam is comparable to that of the fundamental component of the vector soliton.

In the next example shown in Fig.6 the scalar soliton and the lobe of the dipole it interacts with are out-ofphase. Both solitons propagate initially along parallel trajectories. Because of the phase relation the interaction is now repulsive leading to rotation of the dipole. Again the rotation is limited by the anisotropic refractive index distribution. On further propagation, the vector and scalar solitons are clearly repelled and the vector soliton will reorientate to the stable vertical direction once the effect due to presence of the scalar beam is negligible. Our simulations show that by increasing the angle between initial trajectories of both solitons one can induce even stronger rotation of the dipole but this constitutes so drastic perturbation to the dipole that it often breaksup so that the dipole lobes are no longer trapped.

\section{Dipole-dipole scattering}

The next few examples of the numerical simulations involve the collisions of two dipole-mode vector solitons. Unless stated otherwise, the dipole components of the vector solitons are coherent with each other while the fundamental components are mutually incoherent.

In the example depicted in Fig.7 the two identically oriented vector solitons collide centrally propagating along the direction of the applied electric field. The mutual interaction is now attractive (phases of dipoles coincide) and since the intersection angle (or transverse velocities) of the solitons is relatively small the solitons fuse forming a new dipole-mode vector soliton. This fused soliton undergoes internal oscillations as its parameters are quite different from the exact soliton solution. Further simulations show that after emitting some radiation the structure reaches a steady state.

In Fig. 8 the phase of one of the dipoles has been shifted by $\pi$ and hence each lobe now collides with an out of phase lobe of the other dipole. This leads to the mutual repulsion of the dipole-mode vector solitons, which the weak attraction of the incoherent Gaussian beams is unable to counteract. Further simulations show that the structure of each soliton is well preserved throughout the interaction and therefore this collision could be considered as an almost elastic collision. Note very close similarity between this result and simulations of dipoles interaction in isotropic medium shown in Fig.4.

If the initial trajectories of both solitons are chosen such that the collision is non-central then both dipoles usually undergo spatial rotation as shown in Fig.9. This rotation is initiated by the mutual repulsion of the out-ofphase lobes in respective dipole components. Again, the rotation is limited by the anisotropic induced refractive index change as the dipoles force their way past each other. As the solitons pass the zone of interaction they undergo oscillatory rotation about the vertical axis which 
is again due to the orientation perturbation caused by the collision as can be seen in (d),(e) and (f).

Fig.10 shows another example of the noncentral collision of the two dipoles for the case when the initial angle between soliton trajectories is increased to 0.21 degree. As the impact parameter (i.e. lateral mismatch of initial trajectories) is smaller than in the preceding case, the repulsive interaction of dipole components is weaker. This, combined with the larger mutual velocity of the solitons (larger intersection angle) allows the solitons to pass through one another with a weak, but still visible, intermediate tilt of their axes. The final state corresponds to the perturbed dipole states with an excited internal oscillation which is reflected in an unequal energy distribution between both lobes in each dipole.

Finally, Fig.11 shows an example of two dipole-mode vector solitons colliding along their long (y) axes. In this particular example the fundamental components of both dipole solitons are coherent and in-phase. The initial orientation of the dipoles was such that the two directly interacting lobes were out-of-phase hence their interaction was repulsive. On the other hand the interaction of fundamental components is strongly attractive. Since the mutual velocity of the solitons is less (smaller intersection angle) the fundamental components fuse in collision trapping two out-of-phase lobes. In this way new dipole mode vector solitons was formed with its dipole component being constituted by two out-of-phase lobes coming from two different solitons. Notice that because of symmetry of the problem this new vector soliton does not exhibit any transversal motion. The remaining the outmost lobes are no longer bound and separate as fundamental solitary beams as can be seen in Fig.11(e).

\section{EXPERIMENTAL RESULTS}

The generation of an isolated dipole-mode vector soliton was reported earlier in Ref. 4, 19, 20]. Fig.12 shows schematically experimental set-up used in those and current studies.

The dipole-mode soliton can be created using a few different processes: (i) phase imprinting, when one of the beam components is sent through a phase mask in order to imprint the required phase structure, and (ii) symmetry-breaking instability of a vortex-mode composite soliton or (iii) superposition of two oppositely charged vortex beams. In this way, one obtains a dipole-like structure with a phase jump along its transverse direction that is perpendicular to the optical axis of the crystal [see Fig.13(a)]. This dipole-like beam is then combined with the second, node-less beam and the resulting composite beam is focused on the input face of the photorefractive SBN crystal (the crystal has the same parameters as in [4]), biased with a DC field of 1-2.5 $\mathrm{kV}$ applied along its optical axis. The exit and input facets of the crystal are viewed by CCD cameras and images stored in the computer. To control the degree of saturation of the nonlinearity, we illuminated the crystal with a wide beam derived from a white light source. In our experiments the ratio of the peak intensity of the soliton beam to intensity of white light illumination was always of the order of unity. To ensure that both beams forming a composite structure are mutually incoherent, one of them is reflected by a mirror mounted on a piezo-electric transducer $(\mathrm{PZT})$. When the transducer is driven by a $1 \mathrm{kHz}$ signal it introduces a phase modulation into the beam. As the photorefractive crystal is slow, it cannot follow fast changes of the relative phase of both beams making them effectively incoherent. Propagating in a self-focusing saturable medium, such a composite input beam creates a dipole-mode vector soliton, as shown in Fig.13(b-d). As discussed above, the fundamental component creates an effective asymmetric waveguide that guides a dipole-like mode in the form of two out-of-phase solitary beams that otherwise would repel and fly apart.

To observe the soliton-dipole interaction effects, we launched a scalar soliton beam against the dipole soliton. The input state is shown in Fig.13(e), where the dipole-mode soliton is presented by its two-lobe $u$ component only. The relative phase of the soliton and the upper lobe of the dipole is close to $\pi$. Therefore, when the soliton interacts with a dipole, it gets deflected (repulsive interaction) and transforms a part of its linear momentum into an angular momentum of the dipole that starts rotating clockwise which is clearly visible in Fig.13 (f-h). When the scalar soliton is removed, the vector soliton rotates back until it realigns to its original stable orientation orientation which is vertical in the experimental situation discussed here. Fig.14 illustrates the dynamics of this process showing the intensity of both, dipole (top row) and fundamental (bottom row) components. It should be noted that the above experimental observations are in accordance with the results of numerical modelling. In particular, the intensity distributions shown in Fig.13(e-h) closely resemble theoretical results displayed in Fig.1(a) and Fig.6(c) Also, the observed dynamical behavior of the dipole agrees well with our (not shown here) numerical simulations.

In the subsequent graph (Fig.15) we show the interaction of two closely spaced dipole mode vector solitons. These solitons were propagating in parallel. Graphs Fig.15(a-b) shows the intensity of dipole and fundamental components at the input face of the crystal. Fig.15(c) depicts the dipole components of both solitons seen at the exit of the crystal. This picture was obtained by superimposing two graphs corresponding to different solitons, hence it displays propagation without interaction. The dipole-soliton interaction is shown in the bottom row of this figure. Graphs in Fig.15(d-f) show the total intensity (d) and the intensity of dipole (e) and fundamental components of the interacting dipole-mode solitons. Because of $\pi$ phase difference between nearest lobes the interaction leads to a repulsion of the corresponding lobes and the clockwise rotation of both vector solitons. This behavior is analogous to that found in our numerical sim- 
ulations shown in Fig.9(b-c). In both, theory and experiment, the mutual rotation of the interacting dipoles is evident.

\section{CONCLUSIONS}

We have studied interactions of dipole-mode composite solitons with different nonlinear localized structures such as scalar solitons and other dipole-mode solitons. Our studies demonstrate that, apart from the robustness of the dipole-mode solitons against strong perturbations such as the interaction with other solitons (which is a consequence of their linear stability predicted earlier), in many cases the dynamics of the dipole-mode composite solitons can be understood qualitatively as that of the bound states of simpler solitons. Such dynamics involves two major degrees of freedom of the composite soliton, namely, the rotation of the structure as a whole and the relative oscillation of the lobes of the dipole, which both can be excited in collisions. In some cases, the excitation of the dipole soliton is so strong that the dynamics of the composite structure resembles that of a pair of spiraling beams analyzed earlier in Ref. [10]. This is only one of many interesting phenomena observed in our numerical simulations of dipole-mode soliton scattering which also include the excitation of rotational motion by collision with a scalar soliton, annihilation or strong deflection of the incident soliton, etc. Even richer effects are observed when two dipole-mode solitons collide with a nonzero impact parameter. It is remarkable that the rich dynamics observed here may be understood qualitatively in terms of the structure of the colliding objects and the relative phases of the dipole components. To make our results more realistic providing a comparison with experiment, we have extended our analysis and have studied the anisotropic nonlocal model that is more relevant for describing photorefractive nonlinearities. Finally, we have verified some of our theoretical predictions experimentally employing the self-trapping effect in nonlinear photorefractive crystals.

A rich variety of the scattering effects described theoretically and verified experimentally might make the dipole-mode solitons attractive candidates for realistic applications in the field of integrated optics.

\section{Acknowledgements}

This work was partially supported by the Planning and Performance Fund of the Australian National University, the Australian Research Council, the grant BFM2000-0521 (Ministerio de Ciencia y Tecnologa) and the grant PAC02-002 (Consejeria de Ciencia y Tecnologia de la Junta de Comunidades de Castilla-La Mancha), Australian-German Joint Reserach Co-operation Scheme, Australian-German project exchange program by DAAD, and the DFG-Graduiertenkolleg "Nichtlineare kontinuierliche Systeme".
[1] Yu.S. Kivshar and G.P. Agrawal, Optical Solitons: From Fibers to Photonic Crystals (Academic Press, San Diego, 2003), 560 pp.

[2] See an overview by G.I. Stegeman and M. Segev, Science 286, 1518 (1999).

[3] J.J. García-Ripoll, V.M. Pérez-García, E.A. Ostrovskaya, and Yu.S. Kivshar, Phys. Rev. Lett. 85, 82 (2000).

[4] W. Królikowski, E.A. Ostrovskaya, C. Weilnau, M. Geisser, G. McCarthy, Yu.S. Kivshar, C. Denz, and B. Luther-Davies, Phys. Rev. Lett. 85, 1424 (2000).

[5] Z.H. Musslimani, M. Segev, D.N. Christodoulides, and M. Soljacić, Phys. Rev. Lett. 84, 1164 (2000).

[6] Z.H. Musslimani, M. Soljacić, M. Segev, and D.N. Christodoulides, Phys. Rev. E 63, 066608 (2001).

[7] L. Crasovan, Y. V. Kartashov, D. Mihalache, L. Torner, Y. S. Kivshar, and V.M. Pérez-García, Phys. Rev. E 67, 046610 (2003); Y. V. Kartashov, L. Crasovan, D. Mihalache, and L. Torner, Phys. Rev. Lett. 89, 273902 (2002); L. Crasovan, G. Molina-Terriza, J. P. Torres, L. Torner, V.M. Pérez-García, and D. Mihalache, Phys. Rev. E 66, 036612 (2002).

[8] J.P. Reithmaier, M. Röhner, H. Zull, F. Schäfer, A. Forchel, P.A. Knipp, and T.L. Reinecke, Phys. Rev. Lett. 78, 378 (1997).

[9] M. Bayer, T. Gutbrod, J.P. Reithmaier, A. Forchel, T.L. Reinecke, P.A. Knipp, A.A. Dremin, and V.D. Kulakovskii, Phys. Rev. Lett. 81, 2582 (1998).
[10] A. V. Buryak, Yu. S. Kivshar, M. Shih, and M. Segev, Phys. Rev. Lett. 82, 81 (1999), and references therein.

[11] N. Fressengeas, J. Maufoy, and G. Kugel, Phys. Rev. E 54, 6866 (1996).

[12] S. Bian, J. Frejlich, and K.H. Ringhofer, Phys. Rev. Lett. 78, 4035 (1997).

[13] J. Maufoy, N. Fressengeas, D. Wolfersberger, and G. Kugel, Phys. Rev. E 59, 6116 (1999).

[14] M.-F. Shih, C.-C. Jeng, F.-W. Sheu, and C.-Y. Lin, Phys. Rev. Lett. 88, 133902 (2002).

[15] J. Yang and D.E. Pelinovsky, Phys. Rev. E 67, 016608 (2003).

[16] W. Królikowski, M. Saffman, B. Luther-Davies, and C. Denz, Phys. Rev. Lett. 80, 3240 (1998).

[17] See, e.g., A.A. Zozulya and D.Z. Anderson, Phys. Rev. A 51, 1520 (1995); A. Stepken, M.R. Beliĉ, F. Kaiser, W. Królikowski, and B. Luther-Davies, Phys. Rev. Lett. 82, 540 (1999).

[18] D. Neshev, G. McCarthy, W. Królikowski, E. Ostrovskaya, Yu.S. Kivshar, G. Calvo and F. Agullo-Lopez, Opt. Lett. 26, 1185 (2001)

[19] A. Desyatnikov, D. Neshev, E. Ostrovskaya, Y. Kivshar, G. McCarthy, W. Królikowski, B. Luther-Davies. J. Opt. Soc. Am. B 19, 586 (2002).

[20] K. Motzek, F. Kaiser, C. Weilnau, C. Denz, G. McCarthy, W. Królikowski, A. Desyatnikov, and Yu.S. Kivshar, Opt. Commun. 209, 501 (2002). 


\section{Figure Captions}

- Fig.1. Soliton-dipole scattering. (a) Snapshots of the intensity profile of each of the beams taken at few points along their propagation distance. (b) Three-dimensional plot of the total intensity $|u|^{2}+$ $|v|^{2}$, which shows the dipole rotation induced in the collision. (c) Same as (b), but with the $u$ and $v$ components shown separately.

- Fig.2 (a, b) Components of the linear momentum of the incident soliton (solid line) and dipole (marked by circles) after an inelastic collision with a large incident momentum, $\mathbf{p}_{\mathbf{u}} \equiv \int u^{*} \nabla u d \mathbf{r}_{\perp}$, as a function of the impact parameter $d_{y}$, which shows the crucial role of the dipole asymmetry. Total $p_{y}$ does not vanish because of radiation (not seen in the figure).

- Fig.3 Absorption of a soliton by a dipole. (a) Intensity profile of each of the beams at various value of their propagation distance - the darker the more intense; (b) Three-dimensional plot of the total beam intensity; (c) Same as in (b) with the $u$ and $v$ components separated.

- Fig.4 Collisions of two dipoles with the zero [(a), (b), and (d)] and nonzero [(c)] impact parameter, and different orientation of the dipole prior the collision [cf. (a) and (d)].

- Fig.5 Soliton absorption in the anisotropic nonlocal model. The top row shows the dipole components of the vector solitons while the bottom row shows the Gaussian components when a colliding soliton is absorbed by the dipole. Relative intensities: Gaussian $=1.4$, Dipole $=1.1$, Coherent beam $=1.0$. Frames are taken at $z=0.0,1.1,2.6$, 4.1, 5.6 diffraction lengths, respectively. Collision angle $=0.24^{\circ}$. The applied field is in the horizontal direction.

- Fig.6 Soliton-dipole interaction in the anisotropic model. The top row shows (a) initial dipole components, (b) stable orientation of the dipole components with no interacting scalar soliton at $z=2.3$, and (c) rotated dipole when in the presence of a scalar soliton also at $z=2.3$. The scalar soliton is of out of phase with the dipole lobe closest to it. The bottom row shows the corresponding fundamental components. The applied field is in the horizontal direction. Relative intensities: fundamental component $=1.2$, dipole $=1.1$, scalar beam $=1.0$.

- Fig.7 Dipole fusion in the anisotropic model. The top row shows the dipole components of the vector solitons while the bottom row shows the Gaussian components. Relative intensities: fundamental $=1.0$, dipoles $=1.8$. Frames are taken at $z=0.0,3.4,4.8,5.9,7.0$, respectively. Collision angle $=0.13^{\circ}$.
- Fig.8 Elastic collision of two dipoles in the anisotropic model. The top row shows the dipole components of the vector solitons, while the bottom row shows the Gaussian components. Relative intensities: Gaussian=1.0, Dipole=1.8. Frames are taken at $z=0.0,1.5,3.0,4.5,6.0$, respectively. Collision angle $=0.21^{\circ}$.

- Fig.9 Dipole rotation in the anisotropic model. The top row shows the dipole components of the vector solitons while the bottom row shows the Gaussian components. Relative peak intensities: Gaussian=1.0, Dipole=1.2. Frames are taken at $z=0.0,2.3,3.4,4.5,5.6,6.7$, respectively. Collision angle $=0.19^{\circ}$.

- Fig.10 The same as in Fig.9 but for the collision angle $=0.21^{\circ}$. Frames are taken at $z=0.0,2.3,3.0$, $3.7,4.4$, respectively.

- Fig.11 Dipole collision in the anisotropic model. The top row shows the dipole components of the vector solitons while the bottom row shows the fundamental components. Relative intensities: Gaussian $=0.65$, Dipole $=1.1$. Frames are taken at $z=0.0,1.5,3.7,5.2,6.7$, respectively. Collision angle $=0.17^{\circ}$.

- Fig.12 Experimental setup. PM-phase mask, Ffilter, $\lambda / 2$ - half-waveplate, P-polarizer, PBSpolarizing beam splitter, PZTM-mirror mounted on piezo-electric transducer, O- microscope objective, F-filter, V-DC biasing field applied to the crystal, CCD - camera.

- Fig.13 Experimental results. Top row: the formation of the dipole soliton. (a) Initial intensity of the dipole component; (b) total intensity of the vector soliton after $10 \mathrm{~mm}$ propagation in a biased SBN crystal; (c,d) the dipole and fundamental components of the vector soliton after propagation. Bottom row: interaction of dipole and scalar solitons. (e) Initial intensity of the dipole and soliton beams; (f) the same after interaction in a biased SBN crystal; (g-h) dipole and fundamental components of the rotated vector soliton after the interaction. Voltage: $1.3 \mathrm{kV}$. Intensities: dipole- $1.5 \mu \mathrm{W}$, the fundamental component - $0.9 \mu \mathrm{W}$, scalar soliton $1.6 \mu W$.

- Fig.14 Temporal evolution of the dipole component of the tilted vector soliton after the scalar soliton is blocked. (a) initial intensity of the scalar soliton and vector solitons; (b) dipole component immediately after the scalar beam is blocked; (c-g) temporal evolution of the dipole component. The time step between frames is $0.5 \mathrm{sec}$.

- Fig.15 Experimental results for the interaction of two dipoles. Top row: (a-b) intensity distribution 
of the two dipoles and two fundamental components; (c)superimposed images of the dipole components of two independently propagating vector solitons (no interaction); Bottom row: soliton interaction. (d) total intensity distribution of two interacting solitons; (e-f) their dipole and fundamental components. Voltage: $\mathrm{V}=1.1 \mathrm{kV}$. Intensities of both dipoles: 1.5, $1.6 \mu \mathrm{W}$ Intensities of the fundamental components: $1.6,1.7 \mu \mathrm{W}$. 
This figure "Fig1-3.JPG" is available in "JPG" format from: http://arxiv.org/ps/nlin/0306001v1 
This figure "Fig.4-7.JPG" is available in "JPG" format from: http://arxiv.org/ps/nlin/0306001v1 
This figure "Fig.8-11.JPG" is available in "JPG" format from: http://arxiv.org/ps/nlin/0306001v1 
This figure "Fig.12-15.JPG" is available in "JPG" format from: http://arxiv.org/ps/nlin/0306001v1 